OPEN ACCESS

Edited by:

Tolga Cukur,

Bilkent University, Turkey

Reviewed by:

Nicola Filippini,

University of Oxford, United Kingdom

Lihua Qiu,

Second People's Hospital of

Yibin, China

*Correspondence:

Harish RaviPrakash

harishr2001@hotmail.com

Specialty section:

This article was submitted to

Brain Imaging Methods,

a section of the journal

Frontiers in Neuroscience

Received: 14 November 2020

Accepted: 21 January 2021

Published: 19 February 2021

Citation:

RaviPrakash H, Anwar SM,

Biassou NM and Bagci U (2021)

Morphometric and Functional Brain Connectivity Differentiates Chess

Masters From Amateur Players.

Front. Neurosci. 15:629478.

doi: 10.3389/fnins.2021.629478

\section{Morphometric and Functional Brain Connectivity Differentiates Chess Masters From Amateur Players}

\author{
Harish RaviPrakash ${ }^{1 *}$, Syed Muhammad Anwar ${ }^{1,2}$, Nadia M. Biassou ${ }^{3}$ and Ulas Bagci ${ }^{1,4}$ \\ ' Department of Computer Science, Center for Research in Computer Vision, University of Central Florida, Orlando, FL, \\ United States, ${ }^{2}$ Department of Software Engineering, University of Engineering and Technology, Taxila, Pakistan, \\ ${ }^{3}$ Department of Radiology, Clinical Center, National Institutes of Health (NIH), Bethesda, MD, United States, ${ }^{4}$ Department of \\ Radiology, Feinberg School of Medicine, Northwestern University, Chicago, IL, United States
}

A common task in brain image analysis includes diagnosis of a certain medical condition wherein groups of healthy controls and diseased subjects are analyzed and compared. On the other hand, for two groups of healthy participants with different proficiency in a certain skill, a distinctive analysis of the brain function remains a challenging problem. In this study, we develop new computational tools to explore the functional and anatomical differences that could exist between the brain of healthy individuals identified on the basis of different levels of task experience/proficiency. Toward this end, we look at a dataset of amateur and professional chess players, where we utilize resting-state functional magnetic resonance images to generate functional connectivity (FC) information. In addition, we utilize T1-weighted magnetic resonance imaging to estimate morphometric connectivity (MC) information. We combine functional and anatomical features into a new connectivity matrix, which we term as the functional morphometric similarity connectome (FMSC). Since, both the FC and MC information is susceptible to redundancy, the size of this information is reduced using statistical feature selection. We employ off-the-shelf machine learning classifier, support vector machine, for both single- and multi-modality classifications. From our experiments, we establish that the saliency and ventral attention network of the brain is functionally and anatomically different between two groups of healthy subjects (chess players). We argue that, since chess involves many aspects of higher order cognition such as systematic thinking and spatial reasoning and the identified network is task-positive to cognition tasks requiring a response, our results are valid and supporting the feasibility of the proposed computational pipeline. Moreover, we quantitatively validate an existing neuroscience hypothesis that learning a certain skill could cause a change in the brain (functional connectivity and anatomy) and this can be tested via our novel FMSC algorithm.

Keywords: functional connectivity, morphometric similarity, MRI, machine learning, functional morphometric similarity

\section{INTRODUCTION}

Functional connectivity networks (FCNs) are representative of relationships between spatially separated brain regions. The study of FCN, as a technique for diagnosis of clinical conditions, has gained an increase in popularity due to their high test-retest reliability and reproducibility (Zuo and Xing, 2014). There are computational methods proposed to generate FCNs which can delineate 
similarities and differences between healthy controls and diseased subjects (Dosenbach et al., 2010; Chen et al., 2013; Thompson and Apostolova, 2014). While methods that use machine learning approaches for analyzing FCNs have shown great promise (Wee et al., 2012; Chen et al., 2016; He et al., 2018), FCN-based classification methods could suffer from high dimensionality issues where there are more features than the available data. The use of feature selection algorithms with sparse learning is a common approach employed to handle such feature dimensionality problems. Sparse learning techniques, for instance, were successfully applied for diagnosis of Alzheimer's disease (AD) (Casanova et al., 2011), attention deficit hyperactivity disorder (ADHD) (Wang et al., 2015), and epilepsy (Munsell et al., 2015). While there is evidence that FCNbased methods are useful for studying various clinical conditions, these have not yet been widely adapted to analyze two groups of healthy subjects. Structural and functional brain studies have shown that there are structural correlates of intelligence where for intelligent people, the brain regions are known to be more connected (Basten et al., 2015). We propose to extend the applicability of sparse learning methods from diseased subjects to healthy subjects consisting of professional chess players (grand masters) and amateurs.

Skill acquisition, which could include long-term practicing of a particular set of actions, can lead to changes in the brain structure. For instance, in rats trained toward a reaching task with a single fore-paw, an increase in strength of horizontal connections was observed in the motor cortex (Rioult-Pedotti et al., 1998). The effect of long-term skill acquisition on human subjects was studied by evaluating changes in the gray matter volume using voxel-based morphometry (VBM) (Gaser and Schlaug, 2003). In another study, VBM was used to study the morphological changes associated with complexity of navigation induced learning in the brain for London taxi and bus drivers (Maguire et al., 2006). An increase in the midposterior hippocampus gray matter volume was observed in taxi drivers, who tend to remember complex navigation details better, compared to bus drivers. For another example, for a group of professional badminton players, altered functional connectivity patterns were found between the left superior parietal and frontal regions when compared to controls ( $\mathrm{Di}$ et al., 2012). It was hypothesized that even short-term skill acquisition, such as learning to juggle for 3 months, can lead toward detectable changes in the human brain (Driemeyer et al., 2008). A transient increase in gray matter was observed in the occipito-temporal cortex region which comprises the motion sensitive area within the brain. In a more recent study, the effect of mindfulness meditation training in novices identified structural and functional changes in precuneus and posterior default mode network (Yang et al., 2019).

In summary, the research to date has shown clear evidences for structural and functional differences in the brain for two healthy groups of subjects considering a particular skill set. Resting-state functional magnetic resonance images (rs-fMRI) could ideally be suited for studying functional differences by helping in understanding functionally connected regions when the brain is at rest. This can be combined with magnetic resonance imaging (MRI) to extend functional connections to structural connections to identify local and global changes in the brain. We intend to identify what makes a person proficient at a certain skill or what part of the brain (anatomical/functional) is altered by this skill through analyzing the brain images and further contribute toward classifying two groups of healthy subjects in an expert/amateur paradigm by learning discerning features.

\subsection{Summary of Our Contributions}

We adapt sparse learning methods (feature reduction and machine learning algorithms) for the brain analysis of different groups of healthy subjects, where one group consists of professional chess players (with many years of experience) and a second group of amateur subjects. Since chess is a demanding board game where cognitive ability is linked to skilled performance; we hypothesize that there are significant differences, both structurally and in functionally localized brain connections, that could be identified between professional and amateur chess players. To test this hypothesis, we use brain imaging data curated from grand-master level chess players and controls (amateur chess players) (Li et al., 2015). Our major contributions are the following,

- We analyze morphometric measures on the basis of functional dominance by using a functional parcellation network.

- We propose a novel functional morphometric similarity connectome (FMSC) by combining the anatomical and functional information and enabling sparse learning.

- We classify two groups of healthy controls (chess players) based on their skill specialization (professional and amateur) using the proposed FMSC and hence, identify the structural and functional differences between these groups.

\subsection{Related Work}

The precuneus was found to be activated during the perception of chess board patterns (Wan et al., 2011). To compare anatomical regions in the brain of chess masters and amateur players, a voxel-by-voxel volumetric comparison was performed using a two-sample $t$-test (Duan et al., 2012a). A decrease in gray matter volume in the left- and right-caudate regions was found for chess masters compared to amateur controls. Additionally, a resting-state analysis with respect to connections from the caudate also found increased correlations to the posterior cingulate cortex and bilateral angular gyrus. In another study, chess experts demonstrated specific anatomical features in the caudate nucleus, occipito-temporal junction, and the precuneus (Hänggi et al., 2014). A graph theory analysis on rs-fMRI chess data revealed evidence of increased small-world topology and functional connectivity in chess experts compared to amateur players (Duan et al., 2014). The functional connectivity matrices were thresholded at different edge strengths to achieve the desired network sparsity. It can be inferred that differences between chess masters and amateur players could exist in the brain topology and functional organization. However, the influence of global topological changes on functional connectivity and vice-versa is not know for such healthy subjects. One way of developing such 
TABLE 1 | Subject demographics of the chess masters and amateur players dataset.

\begin{tabular}{lccc}
\hline & Chess masters & Amateur players & $\boldsymbol{p}$-value \\
\hline Age & $28.67 \pm 9.06$ & $24.95 \pm 6.14$ & 0.1164 \\
Education & $13.71 \pm 2.64$ & $13.87 \pm 2.64$ & 0.8387 \\
Gender (M/F) & $16 / 8$ & $8 / 15$ & - \\
\hline
\end{tabular}

an understanding would be to study the brain morphology using various non-invasive imaging techniques.

A different approach by Sabuncu et al. (2016) utilized brain morphology to explain phenotypic variations and identify a global statistical association between brain morphology and observable traits. More recently, the morphometric similarity network (MSN) was proposed to map the network architecture of anatomically connected regions in the brain (Seidlitz et al., 2018), where a correlation between morphometric measures and regions of the brain was computed. It was found that MSN modules reiterated known cortical cytoarchitectonic divisions establishing MSN as a valid measure. Structural connectivity can be considered as the basis of functional connectivity and the relationship between these two has been studied in mice (Grandjean et al., 2017), for humans using simulations (Stam et al., 2016) and real data (Uddin, 2013), and for specific tasks such as cognition (Zimmermann et al., 2018). However, there is a limited evidence of work analyzing the combined effects of anatomical and functional differences. In most cases, anatomical differences were identified and used to localize the search for functional differences.

\section{METHODS}

\subsection{Data Preparation}

\subsubsection{Anatomical and Functional Imaging Data}

The subject demographics are as shown in Table 1. All experiments were carried out by following and conforming to the ethical guidelines and the study was approved by research ethics committee of West China hospital of Sichuan university. An informed consent was taken from all participants involved in the study. The anatomical images were acquired using a Siemens 3T TRIO system with a repetition time (TR) of $1,900 \mathrm{~ms}$, echo time (TE) of $2.26 \mathrm{~ms}$, flip angle of $12^{\circ}$. A total of 176 sagittal slices, each with a slice thickness of $1.0 \mathrm{~mm}$ and a voxel size of $1 \times 1 \times 1 \mathrm{~mm}$, were used. The rs-fMRI images were obtained at $\mathrm{TR}=2,000 \mathrm{~ms}, \mathrm{TE}=30 \mathrm{~ms}$, flip angle $=90^{\circ}$. The rsfMRI data comprised of 205 volumes where 30 whole-head axial slices (from each volume), each $5 \mathrm{~mm}$ thick (without gap) and a voxel size of $3.75 \times 3.75 \times 5 \mathrm{~mm}$, were used. The subjects were instructed to relax with their eyes open and visual fixation on a crosshair centered on the screen. More information on the data can be found in the open-source international neuroimaging data-sharing initiative repository (INDI, 2015).

\subsubsection{Anatomical Data Preprocessing}

The mostly used anatomical data preprocessing pipeline was used to support repeatability and reproducibility of our work.
More specifically, and briefly, anatomical features were extracted from high resolution T1-weighted MR images using FreeSurfer (Fischl, 2012). The cortical surface parcellation was performed by following five steps: (1) The MRI volume was registered with the MNI-305 atlas using an affine registration. (2) Bias-field correction and skull stripping were performed. (3) Cutting planes approach was used to remove white- and gray-matter. (4) An initial white surface was generated for each hemisphere which was further refined to follow the intensity gradients between the white- and gray-matter. From this surface, the pial surface was generated by following the intensity gradients between the gray matter and cerebral spinal fluid (CSF). (5) Furthermore, surface labeling was done as in Desikan et al. (2006). The parcellation of the cortex for each subject was based on the 17 network functional parcellation (Thomas Yeo et al., 2011). The metrics of interest, extracted from the cortical parcellations included surface area (SA), gray matter volume (GMV), cortical thickness (CT), curvature index (CI), and folding index (FI). The cortical thickness was measured as the shortest distance from white matter to pial surface. In particular, average cortical thickness $\left(C T_{\text {avg }}\right)$ and standard error $\left(C T_{s d}\right)$ was calculated. The rectified mean curvature $(\mathrm{MC})$ was calculated as,

$$
M C=\frac{\kappa_{1}+\kappa_{2}}{2},
$$

where $\kappa_{1}, \kappa_{2}$ are the maximum and minimum curvatures of the surface. The rectified Gaussian curvature (GC) - an intrinsic measure of the curvature of a surface was measured as,

$$
G C=\kappa_{1} \times \kappa_{2}
$$

The value of CI represented the maximum intrinsic curvature across all points within the surface. The FI value gives a measure of the local gyrification and was computed as,

$$
F I=\left|\kappa_{1}\right| \times\left(\left|\kappa_{1}\right|-\left|\kappa_{2}\right|\right) .
$$

Hence, a total of eight anatomical features were extracted from the T1-weighted MRI data. These metrics have been used in various other studies and shown to be discriminative in general.

\subsection{3. rs-fMRI Data Preprocessing}

The data preprocessing for rs-fMRI has been intensively explored and discussed by Arlot and Celisse (2010). In this work, the opensource AFNI (Analysis of Functional NeuroImages) software was used for extraction of functional connectivity networks (Cox, 1996). The first 5 volumes of the rs-fMRI were discarded to account for the time taken by the tissue to reach steady-state upon application of the magnetic field. Following this, despiking of the signal was done after which slice timing correction was performed to account for the time difference when each slice was acquired. Motion correction was then performed by aligning all volumes to a reference volume to account for the head movement during the course of the data recording. The functional image was then registered with the corresponding anatomical T1-weighted MR image and smoothing was performed to enable better groupwise analysis. The white matter and ventricle maps from the 

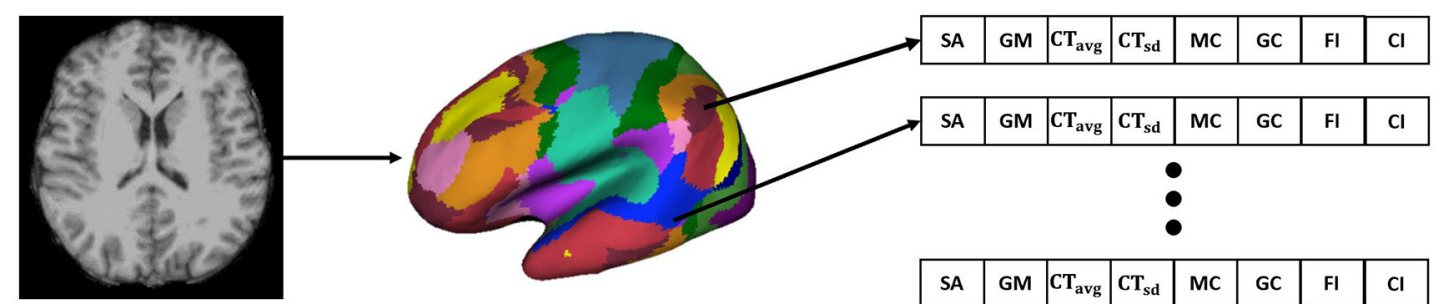

\section{Morphometric measures from each region}

\begin{tabular}{|c|c|c|c|}
\hline SA & Surface area & MC & Mean curvature \\
\hline GM & $\begin{array}{c}\text { Gray matter } \\
\text { volume }\end{array}$ & GC & $\begin{array}{c}\text { Gaussian } \\
\text { curvature }\end{array}$ \\
\hline $\mathbf{C T}_{\text {avg }}$ & $\begin{array}{c}\text { Average cortical } \\
\text { thickness }\end{array}$ & FI & Folding index \\
\hline $\mathbf{C T}_{\text {sd }}$ & $\begin{array}{c}\text { Cortical thickness } \\
\text { standard error }\end{array}$ & $\mathbf{C l}$ & Curvature index \\
\hline
\end{tabular}

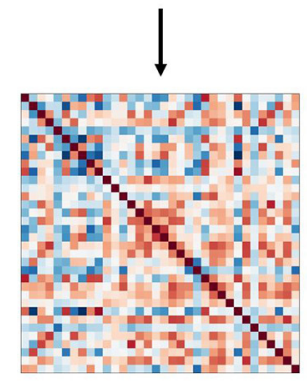

\section{Morphometric Similarity Network (MSN)}

FIGURE 1 | Generating the Morphometric Similarity Network (MSN) from anatomical images where morphometric measures are extracted from the cortical surface of the T1-weighted MRI and Pearson's correlation is used to build the MSN.

FreeSurfer output were used as tissue regressors to detect nonBOLD (blood oxygen level dependent) signals in the data; hence, the final data that we used are considered as "clean." The motion threshold was set to $0.3 \mathrm{~mm}$, with volumes that exceeded this threshold were discarded. Spatial smoothing with a $6 \mathrm{~mm}$ kernel was performed to further reduce the noise and add up coherent signals locally. Finally, band-pass filtering was performed to further isolate the noise with a frequency range of $0.01-0.10 \mathrm{~Hz}$.

\subsection{Morphometric Similarity Network (MSN)}

Morphometric similarity network is a recently proposed method for generating structural connectomes using imaging data from the brain and successfully applied in predicting chronological brain development (Seidlitz et al., 2018). In Seidlitz et al. (2018), the network was generated using diffusion metrics such as fractional anisotropy, mean diffusivity, magnetization transfer and anatomical features including SA, GMV, CT, CI, FI, MC, and GC. For the MSN, the structural connectomes were built using anatomical features. Since correlation requires a feature vector with two or more dimensions, we built several MSNs using combinations of 3 or more anatomical feature vectors. These features have different range of values, e.g., GM and SA values are $>100$, whereas GI, FI, and others have values $<10$. Therefore each feature $(A)$, from a particular region $i$, was $z$-score normalized across all regions as

$$
A_{i}^{z}=\frac{A_{i}-\mu_{A}}{\sigma_{A}} .
$$

The Pearson's correlation between feature vectors of different regions (17 networks) was computed to build each MSN. Since, we used the Yeo-17 network parcellation map (Thomas Yeo et al., 2011), there were 34 regions of interest across both hemispheres, hence the dimension of the generated MSNs was $34 \times 34$. The overall network generation process is illustrated in Figure $\mathbf{1 .}$

\subsection{Functional Connectivity Network (FCN)}

Functional connectivity is defined as the correlation of timeseries between different voxels or different group of voxels. A FCN can be computed from task-based or rs-fMRI data. In rsfMRI, no external stimulus is provided while the BOLD signal is recorded to observe the resting-state of the brain. While in taskbased evaluation, an external stimulus (finger-tapping, listening to story etc.) is established which alternates with a duration of no stimulus in the experimental design paradigm. For this study, the resting-state paradigm was preferred to examine the general functional differences between the two groups. The Yeo17 network parcellation (Thomas Yeo et al., 2011) map was overlaid on the preprocessed rs-fMRI images and the average time-series in each functional parcel was extracted. This step amounts to averaging the time-series across all nodes in the parcel. The Pearson's correlation was then used to compute the correlation between different parcels and generate the functional connectivity network.

\subsection{Proposed Functional Morphometric Similarity Connectome (FMSC)}

We hypothesize that there are both structural and functional differences between the brains of two distinct healthy groups, and that these differences are highly non-linear and hence difficult to capture. Hence, we propose a novel approach to combine the anatomical and functional modalities for indirectly building and identifying this relationship (Figure 2). Toward this, we first extracted morphometric measures: Figure 2-left, and then extracted the FCN. Moreover, since graph metrics enable us to better understand the network topology beyond simple correlation values (Bullmore and Sporns, 2009), we computed the 


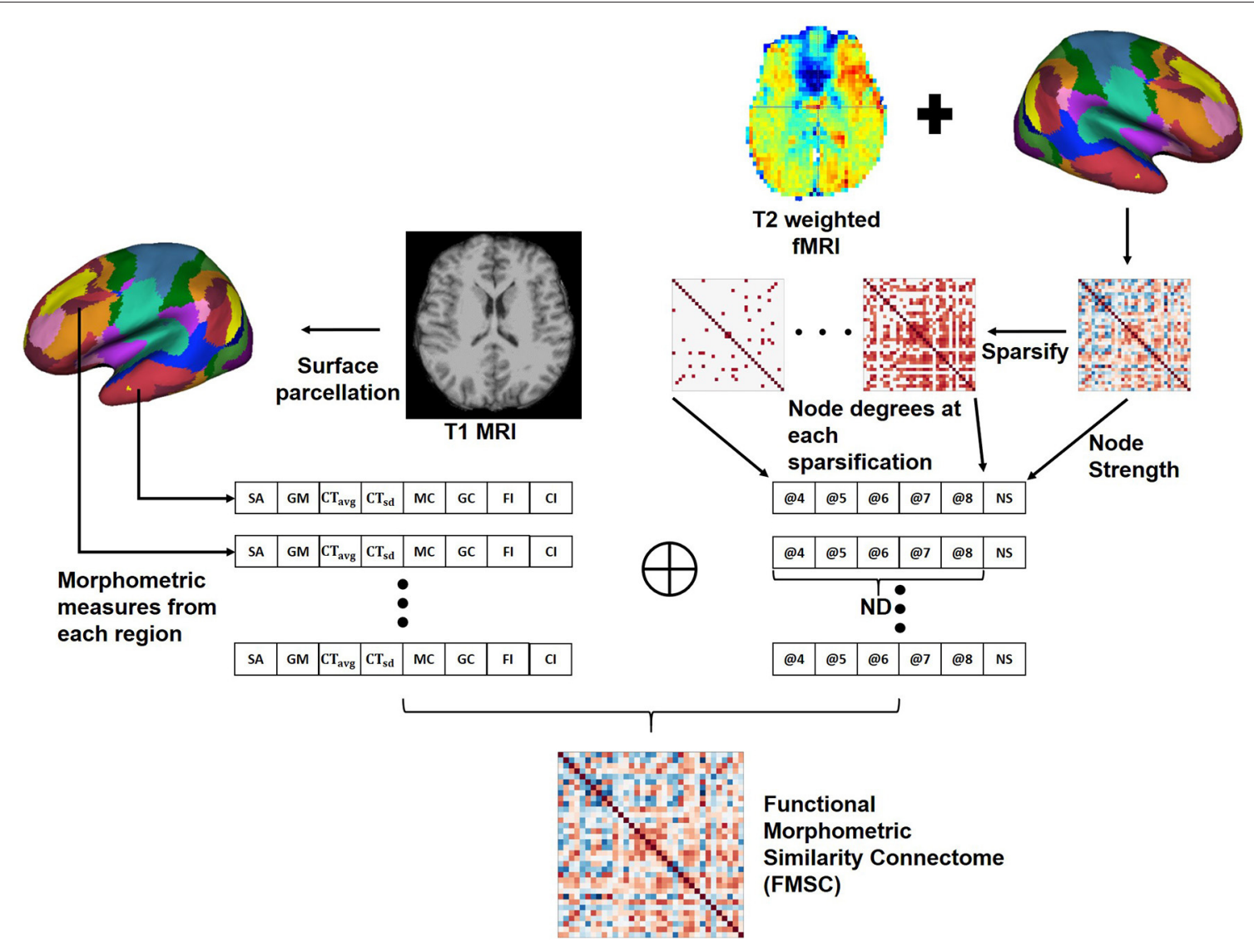

FIGURE 2 | Generating the Functional Morphometric Similarity Connectome (FMSC) from the anatomical and function images. Left: Morphometric measures are extracted from the cortical surface of the T1-weighted MRI. Right: Functional connectivity is generated from the surface of the rs-fMRI and node degrees (ND) are extracted via sparsification (thresholding the edge strength). The morphometric and functional measures are combined to generate the FMSC. NS, node strength; @, thresholded at.

graph metrics-node strength (NS) and node degree (ND), from the FCN. Node degree treats the network as a binary network and represents the number of edges connected to the node which could help in identifying highly connected nodes or hubs in the brain connectome. While node strength, an analog of node degree for weighted networks, represents the sum of the weights of edges connected to the node and helps in identifying the importance of the node.

In particular, we generated sparsified connectivity matrices by thresholding the edge strength of the FCN between $[0.4,0.8]$, at a step size of 0.1 , to successively eliminate weak correlations in the network. This sparsification approach lead to the generation of several undirected connectivity matrices. We then used the ND from these new matrices and the NS from the original FCN as functional measures in place of the Pearson's correlation (Figure 2-right). Finally, we combined the morphometric and functional measures into a single node feature vector which was normalized using the $z$-score (Equation 1). The correlation between different nodes was computed using this feature vector to build the novel connectivity matrix, the functional morphometric similarity connectome.

\section{RESULTS}

\subsection{Statistical Analysis}

To identify whether morphometric measures (derived from functionally defined anatomical regions) are distinct between chess masters and amateur players, we performed a two-sample $t$-test for each brain region and metric (see Figure 1). The significance value was set at $p<0.05$, and a family-wise error (FWE) correction based on the Holm-Sidak method (Šidák, 1967) was employed to control the number of Type-I errors, while also reducing the increased risk (due to correction) of Type-II errors. Two significant regions were identified along with cortical thickness as the metric of interest. Table 2 shows the regions and the FWE corrected $p$-values. It should be noted that these regions (somatomotor $\mathrm{A}$ and peripheral visual) are different from those previously identified in literature, which shows the benefit of using functional parcellation. Since the chosen parcellation comprises of spatially non-contiguous brain regions, we performed the $t$-test with the anatomical parcellation atlas-Destrieux atlas (Destrieux et al., 2010), which has 74 gyral and sulcal regions. Among these regions, six regions were found statistically significantly different between groups and are 
TABLE 2 | Anatomically significant functionally defined regions and the corresponding metric of difference.

\begin{tabular}{lccc}
\hline ROI & Metric & Hemisphere & p-value* \\
\hline Yeo-17 network parcellation & & & \\
Somatomotor A & $C T_{\text {sd }}$ & $\mathrm{L}$ & 0.0443 \\
Peripheral visual & $C T_{\text {avg }}$ & $\mathrm{R}$ & 0.0137 \\
\hline Destrieux atlas & & & \\
Parietal inferior supramarginal G & $C T_{s d}$ & $\mathrm{~L}$ & 0.0494 \\
Precentral G & $G M V$ & $\mathrm{R}$ & 0.0491 \\
Rectus G & $C T_{s d}$ & $\mathrm{R}$ & 0.0094 \\
Rectus G & $G C$ & $\mathrm{R}$ & 0.0053 \\
Rectus G & $C l$ & $\mathrm{R}$ & 0.0135 \\
Central S & $C l$ & $\mathrm{R}$ & 0.0397
\end{tabular}

*FWE corrected; L, Left; R, Right; G, Gyrus; S, Sulcus; CT sd, standard error of cortical

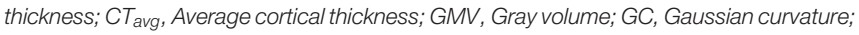
$\mathrm{Cl}$, Curvature Index; $\mathrm{ROI}$, region of interest.

shown in Table 2. These six regions are known to be part of the somatosensory (pre-central gyrus, central sulcus, and supramarginal gyrus) and central execution (gyrus rectus) networks. This is in agreement with neuroscience studies that have shown that highly complex games, such as chess, favors the development of the frontal lobe (Ortiz-Pulido et al., 2019).

\subsection{Classification}

After identifying statistically significantly different regions, we generated the connectivity matrix to identify further differences between two healthy groups of individuals. To ensure a strong classification model, the $k$-fold cross-validation approach Arlot and Celisse, 2010 was used with the value of $k=10$. Note that cross-validation is an approach to do out-of-sample testing with limited data wherein the data is split into training and testing sets to enable model validation. In $k$ - fold cross-validation, the data is split into $k$ different training and test sets and the average performance across all metrics is the overall model evaluation. We used a stratified 10-fold cross-validation technique, where the data splitting ensures that there are a similar number of samples from all classes in the test set.

All connectivity matrices/connectomes generated were symmetrical i.e., they were identical across the diagonal. The diagonal itself represented self-correlation and was always equal to 1. Thus, only the upper-right triangle was extracted from these connectivity matrices and the resulting feature vector was used in the classification task. For an $m \times m$ matrix the feature vector dimension was computed as $\frac{m \times(m-1)}{2}$. Since the networks were $34 \times 34$, the extracted feature vector was of dimension 561. The feature dimension was greater than the size of the training data and thus, feature selection was used to reduce the feature vector size. Toward this, a simple student's $t$-test was performed and only significant features (at a confidence interval of $p<0.05)$ were chosen. The selected features were then used to train a support vector machine (SVM) (Cortes and Vapnik, 1995) with a linear kernel, regularization and scaling parameters were set to $C=1$ and $\gamma=1 e-4$, respectively. Note that
TABLE 3 | Ten-fold cross-validation performance using functional connectivity features.

\begin{tabular}{lccc}
\hline Approach & Accuracy (\%) & Precision (\%) & F1-score (\%) \\
\hline w/o $t$-test & 64.83 & 62.67 & 64.83 \\
w $\boldsymbol{t}$-test & $\mathbf{7 6 . 3 3}$ & $\mathbf{7 6 . 8 3}$ & $\mathbf{7 4 . 0 5}$ \\
\hline
\end{tabular}

The bold values show that the performance improved in all matrices using feature selection. w/o, without; w, with.

any other off-the-shelf classifier can be used for this purpose (including deep learning classifiers), however, the classification performance of SVM is at par. The performance was evaluated using accuracy, precision, and recall. These evaluation metrics are commonly used and widely known in the literature; however, to make the manuscript self-contained, we briefly summarize them as follows.

Accuracy is defined as the ratio of the total number of correctly classified items to the total number of items and was computed as

$$
\text { Accuracy }=\frac{T P+T N}{T P+F P+T N+F N},
$$

where $\mathrm{TP}$-true positives, FP-false positives, $\mathrm{TN}$-true negatives, and FN-false negatives. Precision is defined as the ratio of correctly predicted positive results to the total predicted positive results and was computed as

$$
\text { Precision }=\frac{T P}{T P+F P} .
$$

F1-score is defined as the harmonic mean of precision and recall, where recall is the proportion of correctly predicted positive results, and was computed as

$$
F 1=2 \cdot \frac{\text { precision } \times \text { recall }}{\text { precision }+ \text { recall }}
$$

\subsubsection{FCN-Based Classification}

The effect of feature selection on FCN-based classification was evaluated by first performing a 10 -fold cross-validation using all the extracted features. As a result, SVM was trained using 561 features, resulting in the classification performance presented in Table 3. Later, feature selection (via $t$-test) was performed prior to training the SVM in each fold. This resulted in an average accuracy of $76.33 \%$, a significant improvement (of over $11 \%$ ) compared to the baseline. The common significant connections across all 10-folds of the cross-validation were identified and are shown in Figure 3A. Lateral and medial views show the intrahemispheric connections and inter-hemispheric connections are presented in the dorsal view.

\subsubsection{MSN-Based Classification}

Having established the benefit of using feature selection toward the classification task and our baseline classification accuracy, we performed classification using connectivity features from the MSN networks. Since, we consider the construction of MSNs 

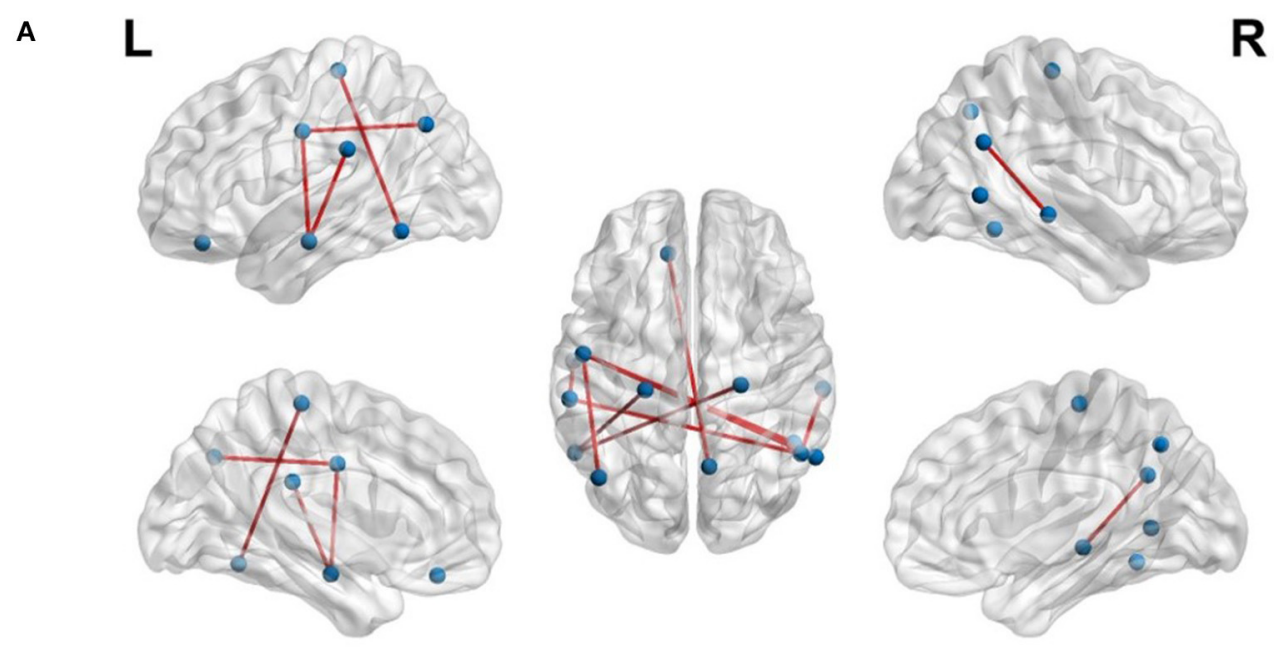

$\mathbf{R}$

B

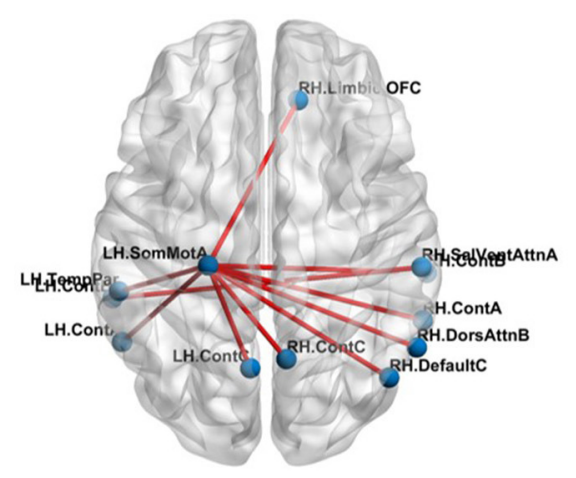

FIGURE 3 | Top-10 connections identified in classification of chess masters vs. amateur players. (A) Functional connections. (B) Morphometric connections. Table 5 lists the names of the ROls in these top connections.

using 3 or more measures, the total number of MSNs was computed as

$$
\text { MSNs }=\sum_{k=3}^{n=8}\left(\begin{array}{l}
n \\
k
\end{array}\right) \text {, }
$$

where the total number of morphometric measures was 8 . There were a total of 219 MSNs and thus, 219 trained SVM models. Among these models, the best 10-fold classification accuracy was achieved using $\mathrm{SA}, \mathrm{CT}_{\mathrm{sd}}$ and $\mathrm{CI}$ as the morphometric features of interest. The MSN representing the connections for this particular model is shown in Figure 3B. We found that the saliency and ventral attention network in the left hemisphere and the dorsal attention network in the right hemisphere had significantly different connections across networks.

\subsubsection{FMSC-Based Classification}

We validated our hypothesis that MSN- and FCN-based approaches contribute complementary information using a simple majority voting strategy, called pseudo-FMSC, combining the best performing MSN- and FCN-based classification models. The common connections across the majority voting model are shown in Figure 4A. The saliency and ventral attention network, dorsal attention network, and central visual network were common across the models. The resulting model had a 6 and 3\% improvement in classification performance over the best performing MSN and FCN models, respectively, reaching an overall accuracy of $80 \%$. This indicated the presence of potentially complementary information in the MSN to that of FCN. Thus, we combined the MSN and FCN metrics via feature concatenation. The FCN was thresholded at edge strengths of $[0.4,0.5,0.6,0.7,0.8]$ and NDs were computed for each of these sparse un-directed networks, whereas NS was computed directly from the FCN. The total number of features was now reduced to 14 (8 morphometric measures and 6 functional measures). Similar to MSN-based classification, we tested all possible combinations and this resulted in $364\left(\left(\begin{array}{c}14 \\ 3\end{array}\right)\right)$ different models. The highest accuracy of $88 \%$ was achieved and Figure $4 \mathrm{~B}$ shows the common significant connections across the 10 -fold cross-validation in the best performing FMSC model.

\section{DISCUSSION}

In this work, we have proposed a novel approach (FMSC) for combining metrics from anatomical and functional brain 


\section{A}

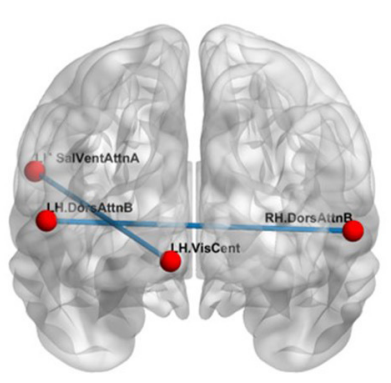

B

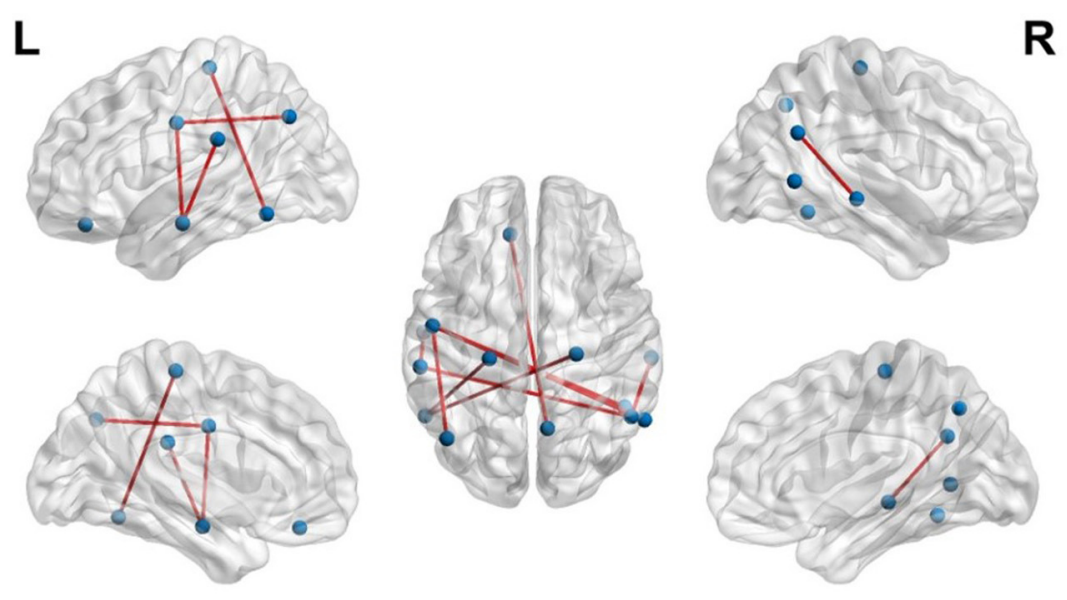

FIGURE 4 | Functional anatomical connections differentiating chess masters from amateur players. (A) Common functional and morphometric connections. (B) Functional-morphometric connections. Table $\mathbf{5}$ lists the names of the ROls in these top connections.

images, to identify distinct connectomes between two groups of healthy subjects, specifically chess masters and amateur players. Our trained machine learning-based model achieved a classification accuracy of $88 \%$ showing drastic improvements over the standard functional connectivity-based approach. The performance measures for the top performing models are presented in Table 4. It should be noted that the best model (using sparsification-based FMSC) significantly improves the performance (accuracy) by $\approx 14 \%$ when compared with MSNbased approach, and $\approx 11 \%$ when compared with FCN-based approach. These results add credence to the success of the proposed FMSC-based classification.

Additionally, we have used functional parcellation that combines functionally coupled regions into the same networks. This allowed us to study whether broadly defined functional networks show morphometric differences. We identified two such coupled regions with statistically significant differences in cortical thickness. The network analysis using the functional parcellation enabled us to look at higher-order connectivity across anatomical regions which are non-local, owing to the functionally defined parcellation. We analyzed the use of morphometric similarity networks toward the classification and found an accuracy above that of a simple coin flip. This further indicates that there are anatomical differences between nonlocal regions and that the relationship may not be a simple linear relationship.
TABLE 4 | Classification results of morphometric similarity networks (MSN), functional connectivity networks (FCN) and functional morphometric similarity (FMSC) on 10-fold cross-validation.

\begin{tabular}{|c|c|c|c|c|}
\hline Approach & Model & $\begin{array}{c}\text { Accuracy } \\
(\%)\end{array}$ & $\begin{array}{l}\text { Precision } \\
\text { (\%) }\end{array}$ & $\begin{array}{c}\text { F1-score } \\
(\%)\end{array}$ \\
\hline MSN & $S A+C T_{S d}+C l$ & 73.83 & 77.5 & 70.92 \\
\hline MSN & $S A+C T_{s d}+F l+C l$ & 71.83 & 73.5 & 69.33 \\
\hline FCN & FCN & 76.33 & 76.83 & 74.05 \\
\hline Pseudo-FMSC & Majority voting & 80.17 & 84.67 & 79.33 \\
\hline FMSC & $\begin{array}{l}\mathrm{CT}_{\text {avg }}+\mathrm{GC}+\mathrm{Fl}+\mathrm{Cl}+ \\
\mathrm{ND} 0.4+\mathrm{ND} @ 0.8\end{array}$ & 87.17 & 91.17 & 86.57 \\
\hline FMSC & $\begin{array}{l}C T_{s d}+\mathrm{GC}+\mathrm{Fl}+\mathrm{Cl}+ \\
\mathrm{ND} 00.6+\mathrm{ND} @ 0.8\end{array}$ & 88.00 & 91.58 & 87.52 \\
\hline
\end{tabular}

SA, Surface area; CT_avg, Average cortical thickness; CT_sd, Cortical thickness standard error; Fl, Folding index; Cl, Curvature Index; GC, Gaussian curvature; ND@, Node degree at threshold level.

The bold values represent the highest values on each of the metrics.

The proposed FMSC-based classification shows that there exists a complex functional-morphometric relationship. To establish whether functional-morphometric measures provide a unique look into the organization, we examined the overlap of top connections between the best MSN, FCN, and FMSC models. The metrics used in each of these networks were different; 
TABLE 5 | Top-10 significant connections based on morphometery, functionality, and functional-morphometery.

\begin{tabular}{|c|c|c|}
\hline Connectome & ROI-1 & ROI-2 \\
\hline \multirow{10}{*}{$\begin{array}{l}\text { Morphometric similarity } \\
\text { network }\end{array}$} & Somatomotor A (L) & Control A (L) \\
\hline & Control B (L) & $\begin{array}{l}\text { Saliency and ventral } \\
\text { attention } A(R)\end{array}$ \\
\hline & Somatomotor A (L) & Control C (L) \\
\hline & Somatomotor A (L) & Tempoparietal (L) \\
\hline & Somatomotor A (L) & Dorsal Attention B (R) \\
\hline & Somatomotor A (L) & Control A (R) \\
\hline & Somatomotor A (L) & Limbic B (R) \\
\hline & Somatomotor A (L) & Control B (R) \\
\hline & Somatomotor A (L) & Control C (R) \\
\hline & Somatomotor A (L) & Default Mode C (R) \\
\hline \multirow{10}{*}{$\begin{array}{l}\text { Functional connectivity } \\
\text { network }\end{array}$} & Peripheral visual (L) & $\begin{array}{l}\text { Saliency and ventral } \\
\text { attention } A(L)\end{array}$ \\
\hline & Dorsal attention B (L) & $\begin{array}{l}\text { Saliency and ventral } \\
\text { attention } A(L)\end{array}$ \\
\hline & Somatomotor B (L) & Control C (L) \\
\hline & Somatomotor B (L) & Default mode A (R) \\
\hline & Somatomotor B (L) & Dorsal attention $B(R)$ \\
\hline & $\begin{array}{l}\text { Saliency and ventral } \\
\text { attention } B(L)\end{array}$ & Control C (R) \\
\hline & $\begin{array}{l}\text { Saliency and ventral } \\
\text { attention } A(L)\end{array}$ & Default mode A (R) \\
\hline & $\begin{array}{l}\text { Saliency and ventral } \\
\text { attention } A(L)\end{array}$ & Dorsal attention B (R) \\
\hline & $\begin{array}{l}\text { Saliency and ventral } \\
\text { attention } A(L)\end{array}$ & Control C (R) \\
\hline & Tempoparietal (L) & Control C (R) \\
\hline \multirow{10}{*}{$\begin{array}{l}\text { Functional morphometric } \\
\text { similarity connectome }\end{array}$} & Default mode A (L) & Somatomotor B (L) \\
\hline & Control A (L) & Somatomotor A (L) \\
\hline & Control A (L) & Somatomotor A (R) \\
\hline & Limbic B (L) & Control C (R) \\
\hline & Default mode B (L) & Somatomotor B (L) \\
\hline & Default mode B (L) & $\begin{array}{l}\text { Saliency and ventral } \\
\text { attention }(\mathrm{L})\end{array}$ \\
\hline & Somatomotor B (L) & Dorsal attention $B(R)$ \\
\hline & Somatomotor B (L) & Dorsal attention $B(A)$ \\
\hline & $\begin{array}{l}\text { Saliency and ventral } \\
\text { attention } A(L)\end{array}$ & Dorsal attention $B(R)$ \\
\hline & Default mode A (R) & Default mode B (R) \\
\hline
\end{tabular}

ROI, Region of Interest; L, Left; R, Right.

therefore; we expected very few regions to be common. We identified an atypical within- and between-hemisphere dorsal attention network (DAN) connection. Since DAN plays a key role in visuo-spatial attention and hence the differences borne from the long-term training in a spatially oriented game, such as chess (Atherton et al., 2003), suggests an increased visuospatial intelligence. It should be noted that DAN is a task-positive network that is cued during externally directed attention tasks (Spreng et al., 2010). We also studied the top-10 connections in the MSN-, FCN-, and FMSC-based classification and are presented in Table 5. The saliency and ventral attention (SVA) network was identified to be in the top-10 connections in both the FCN and FMSC-based approaches, serving as a kind of hub to connections that were significantly different between chess masters and amateur players. The SVA network commonly serves as a switch between the default mode network and the central execution network (Goulden et al., 2014). The betweenhemisphere connection of the SVA and the control network was identified to be functionally different. We also found that the right hemisphere control network is common in both FCNbased and FMSC-based networks as a kind of hub. The default mode network (DMN), in particular default mode A, was identified in both FCN- and FMS-based approaches. The DMN was identified in a previous study showing increased functional connectivity in chess masters (Duan et al., 2012b; Wang et al., 2018). The precuneus, which is a core region in the DMN, was also previously identified to be important in chess (Wan et al., 2011). A minimal overlap between the significant connections across different approaches validates the hypothesis that there are indirect and non-linear relationships between anatomy and function. This indirect relationship can be identified using the proposed FMSC and potentially help in an early diagnosis of several neurological disorders.

There are a few limitations to the proposed approach and analysis. The relatively large voxel volume results in a low signal to noise ratio and thus limits the ability to study small brain regions. Existing studies show that local functional connectivity density differences may exist between women and men (Tomasi and Volkow, 2012). This may be true for differences in processing efficiency in the motor mental rotation task (Christova et al., 2008). In our study, corrections for gender were not performed in the current analysis due to lack of enough statistical power analysis. Although, in a subset of our experiments, we observed that with a gender matched dataset (resulting in fewer subjects), the performance of FCN- and MSN-based methods dropped drastically. But our proposed FMSC-based method continues performing reasonably using different feature combinations even with limited data. We also noted that, by reducing the size of the dataset, the statistical power of the analysis was reduced. Hence gender could make a difference in the identified top significant connections, but the way the data was collected, we could only employ indirect ways to address this and 10 -fold cross-validation is good for that. We will study gender as a biological covariate in our future work to establish fundamental differences between the chess masters and amateur players by using a well-matched larger cohort. In future work, we will use a further splitting of the network labels to analyze morphometric features of individual regions within the functional cluster. Moreover, with the increased dimensionality of the feature vectors, deep learning algorithms can be utilized to perform classification but the size of the dataset may prove to be a limiting factor (RaviPrakash et al., 2019). While MSN enabled us to combine structural information, as a potential extension of this work, we would combine structural information from diffusion tensor imaging based measures such as fractional anisotropy, mean diffusivity, 
and structural node degree into the morphometric measures to further strengthen the functional-morphometric relationship.

\section{DATA AVAILABILITY STATEMENT}

Publicly available datasets were analyzed in this study. This data can be found here: http://fcon_1000.projects.nitrc.org/indi/pro/ wchsu_li_index.html.

\section{ETHICS STATEMENT}

The studies involving human participants were reviewed and approved by The Research Ethics Committee of West China Hospital of Sichuan University. Written informed consent to

\section{REFERENCES}

Arlot, S., and Celisse, A. (2010). A survey of cross-validation procedures for model selection. Stat. Surv. 4, 40-79. doi: 10.1214/09-SS054

Atherton, M., Zhuang, J., Bart, W. M., Hu, X., and He, S. (2003). A functional MRI study of high-level cognition. I. The game of chess. Cogn. Brain Res. 16, 26-31. doi: 10.1016/S0926-6410(02)00207-0

Basten, U., Hilger, K., and Fiebach, C. J. (2015). Where smart brains are different: a quantitative meta-analysis of functional and structural brain imaging studies on intelligence. Intelligence 51, 10-27. doi: 10.1016/j.intell.2015.04.009

Bullmore, E., and Sporns, O. (2009). Complex brain networks: graph theoretical analysis of structural and functional systems. Nat. Rev. Neurosci. 10, 186-198. doi: 10.1038/nrn2575

Casanova, R., Wagner, B., Whitlow, C. T., Williamson, J. D., Shumaker, S. A., Maldjian, J. A., et al. (2011). High dimensional classification of structural MRI Alzheimer's disease data based on large scale regularization. Front. Neuroinform. 5:22. doi: 10.3389/fninf.2011.00022

Chen, G., Saad, Z. S., Britton, J. C., Pine, D. S., and Cox, R. W. (2013). Linear mixed-effects modeling approach to fMRI group analysis. Neuroimage 73, 176-190. doi: 10.1016/j.neuroimage.2013.01.047

Chen, X., Zhang, H., Gao, Y., Wee, C.-Y., Li, G., Shen, D., et al. (2016). High-order resting-state functional connectivity network for mci classification. Hum. Brain Mapp. 37, 3282-3296. doi: 10.1002/hbm.23240

Christova, P. S., Lewis, S. M., Tagaris, G. A., Uğurbil, K., and Georgopoulos, A. P. (2008). A voxel-by-voxel parametric fMRI study of motor mental rotation: hemispheric specialization and gender differences in neural processing efficiency. Exp. Brain Res. 189, 79-90. doi: 10.1007/s00221-008-1405-x

Cortes, C., and Vapnik, V. (1995). Support-vector networks. Mach. Learn. 20, 273-297. doi: 10.1007/BF00994018

Cox, R. W. (1996). AFNI: software for analysis and visualization of functional magnetic resonance neuroimages. Comput. Biomed. Res. 29, 162-173. doi: 10.1006/cbmr.1996.0014

Desikan, R. S., Ségonne, F., Fischl, B., Quinn, B. T., Dickerson, B. C., Blacker, D., et al. (2006). An automated labeling system for subdividing the human cerebral cortex on MRI scans into gyral based regions of interest. Neuroimage 31, 968-980. doi: 10.1016/j.neuroimage.2006.01.021

Destrieux, C., Fischl, B., Dale, A., and Halgren, E. (2010). Automatic parcellation of human cortical gyri and sulci using standard anatomical nomenclature. Neuroimage 53, 1-15. doi: 10.1016/j.neuroimage.2010.06.010

Di, X., Zhu, S., Jin, H., Wang, P., Ye, Z., Zhou, K., et al. (2012). Altered resting brain function and structure in professional badminton players. Brain Connect. 2, 225-233. doi: 10.1089/brain.2011.0050

Dosenbach, N. U., Nardos, B., Cohen, A. L., Fair, D. A., Power, J. D., Church, J. A., et al. (2010). Prediction of individual brain maturity using fMRI. Science 329, 1358-1361. doi: 10.1126/science.1194144

Driemeyer, J., Boyke, J., Gaser, C., Büchel, C., and May, A. (2008). Changes in gray matter induced by learning-revisited. PLoS ONE 3:e2669. doi: 10.1371/journal.pone.0002669 participate in this study was provided by the participants' legal guardian/next of kin.

\section{AUTHOR CONTRIBUTIONS}

All authors significantly contributed to the design, analysis, and evaluation of the proposed method and results. All authors reviewed the manuscript and agreed on the final version.

\section{FUNDING}

This work was partially supported by grants from the NIH' National Cancer Institute (R01CA246704 and R01CA240639).

Duan, X., He, S., Liao, W., Liang, D., Qiu, L., Wei, L., et al. (2012a). Reduced caudate volume and enhanced striatal-dmn integration in chess experts. Neuroimage 60, 1280-1286. doi: 10.1016/j.neuroimage.2012.01.047

Duan, X., Liao, W., Liang, D., Qiu, L., Gao, Q., Liu, C., et al. (2012b). Large-scale brain networks in board game experts: insights from a domain-related task and task-free resting state. PLoS ONE 7:e32532. doi: 10.1371/journal.pone.0032532

Duan, X., Long, Z., Chen, H., Liang, D., Qiu, L., Huang, X., et al. (2014). Functional organization of intrinsic connectivity networks in chinese-chess experts. Brain Res. 1558, 33-43. doi: 10.1016/j.brainres.2014.02.033

Fischl, B. (2012). Freesurfer. Neuroimage 62, 774-781. doi: 10.1016/j.neuroimage.2012.01.021

Gaser, C., and Schlaug, G. (2003). Brain structures differ between musicians and non-musicians. J. Neurosci. 23, 9240-9245. doi: 10.1523/JNEUROSCI.23-27-09240.2003

Goulden, N., Khusnulina, A., Davis, N. J., Bracewell, R. M., Bokde, A. L., McNulty, J. P., et al. (2014). The salience network is responsible for switching between the default mode network and the central executive network: replication from dcm. Neuroimage 99, 180-190. doi: 10.1016/j.neuroimage.2014.05.052

Grandjean, J., Zerbi, V., Balsters, J. H., Wenderoth, N., and Rudin, M. (2017). Structural basis of large-scale functional connectivity in the mouse. J. Neurosci. 37, 8092-8101. doi: 10.1523/JNEUROSCI.0438-17.2017

Hänggi, J., Brütsch, K., Siegel, A. M., and Jäncke, L. (2014). The architecture of the chess player's brain. Neuropsychologia 62, 152-162. doi: 10.1016/j.neuropsychologia.2014.07.019

He, T., Kong, R., Holmes, A. J., Sabuncu, M. R., Eickhoff, S. B., Bzdok, D., et al. (2018). "Is deep learning better than kernel regression for functional connectivity prediction of fluid intelligence?" in 2018 International Workshop on Pattern Recognition in Neuroimaging (PRNI) (Singapore: IEEE), 1-4. doi: 10.1109/PRNI.2018.8423958

INDI (2015). A Multimodal MRI Dataset of Professional Chess Players.

Li, K., Jiang, J., Qiu, L., Yang, X., Huang, X., Lui, S., et al. (2015). A multimodal MRI dataset of professional chess players. Sci. Data 2:150044. doi: 10.1038/sdata.2015.44

Maguire, E. A., Woollett, K., and Spiers, H. J. (2006). London taxi drivers and bus drivers: a structural MRI and neuropsychological analysis. Hippocampus 16, 1091-1101. doi: 10.1002/hipo.20233

Munsell, B. C., Wee, C.-Y., Keller, S. S., Weber, B., Elger, C., da Silva, L. A. T., et al. (2015). Evaluation of machine learning algorithms for treatment outcome prediction in patients with epilepsy based on structural connectome data. Neuroimage 118, 219-230. doi: 10.1016/j.neuroimage.2015.06.008

Ortiz-Pulido, R., Ortiz-Pulido, R., Garcia-Hernandez, L. I., Pérez-Estudillo, C. A., and Ramirez-Ortega, M. L. (2019). Neuroscientific evidence support that chess improves academic performance in school. Revista Mexicana de Neurociencia 20, 194-199. doi: 10.24875/RMN.M19000060

RaviPrakash, H., Watane, A., Jambawalikar, S., and Bagci, U. (2019). “Deep learning for functional brain connectivity: are we there yet?" in Deep Learning and Convolutional Neural Networks for Medical Imaging and Clinical Informatics (Springer), 347-365. doi: 10.1007/978-3-030-13969-8_17 
Rioult-Pedotti, M.-S., Friedman, D., Hess, G., and Donoghue, J. P. (1998). Strengthening of horizontal cortical connections following skill learning. Nat. Neurosci. 1, 230-234. doi: 10.1038/678

Sabuncu, M. R., Ge, T., Holmes, A. J., Smoller, J. W., Buckner, R. L., Fischl, B., et al. (2016). Morphometricity as a measure of the neuroanatomical signature of a trait. Proc. Natl. Acad. Sci. U.S.A. 113, E5749-E5756. doi: 10.1073/pnas.1604378113

Seidlitz, J., Váša, F., Shinn, M., Romero-Garcia, R., Whitaker, K. J., Vértes, P. E., et al. (2018). Morphometric similarity networks detect microscale cortical organization and predict inter-individual cognitive variation. Neuron 97, 231-247. doi: 10.1016/j.neuron.2017.11.039

Šidák, Z. (1967). Rectangular confidence regions for the means of multivariate normal distributions. J. Am. Stat. Assoc. 62, 626-633. doi: 10.1080/01621459.1967.10482935

Spreng, R. N., Stevens, W. D., Chamberlain, J. P., Gilmore, A. W., and Schacter, D. L. (2010). Default network activity, coupled with the frontoparietal control network, supports goal-directed cognition. Neuroimage 53, 303-317. doi: 10.1016/j.neuroimage.2010.06.016

Stam, C., Van Straaten, E., Van Dellen, E., Tewarie, P., Gong, G., Hillebrand, A., et al. (2016). The relation between structural and functional connectivity patterns in complex brain networks. Int. J. Psychophysiol. 103, 149-160. doi: 10.1016/j.ijpsycho.2015.02.011

Thomas Yeo, B., Krienen, F. M., Sepulcre, J., Sabuncu, M. R., Lashkari, D., Hollinshead, M., et al. (2011). The organization of the human cerebral cortex estimated by intrinsic functional connectivity. J. Neurophysiol. 106, 1125-1165. doi: $10.1152 /$ jn.00338.2011

Thompson, P., and Apostolova, L. (2014). Computational anatomical methods as applied to ageing and dementia. Brit. J. Radiol. 80, S78-S91. doi: 10.1259/BJR/20005470

Tomasi, D., and Volkow, N. D. (2012). Gender differences in brain functional connectivity density. Hum. Brain Mapp. 33, 849-860. doi: 10.1002/hbm.21252

Uddin, L. Q. (2013). Complex relationships between structural and functional brain connectivity. Trends Cogn. Sci. 17, 600-602. doi: 10.1016/j.tics.2013.09.011

Wan, X., Nakatani, H., Ueno, K., Asamizuya, T., Cheng, K., and Tanaka, K. (2011). The neural basis of intuitive best next-move generation in board game experts. Science 331, 341-346. doi: 10.1126/science.11 94732

Wang, L., Xu, J., Wang, C., and Wang, J. (2018). Whole brain functional connectivity pattern homogeneity mapping. Front. Hum. Neurosci. 12:164. doi: 10.3389/fnhum.2018.00164

Wang, Y., Li, Z., Wang, Y., Wang, X., Zheng, J., Duan, X., et al. (2015). A novel approach for stable selection of informative redundant features from high dimensional fMRI data. arXiv [Preprint]. arXiv:1506. 08301.

Wee, C.-Y., Yap, P.-T., Zhang, D., Denny, K., Browndyke, J. N., Potter, G. G., et al. (2012). Identification of mci individuals using structural and functional connectivity networks. Neuroimage 59, 2045-2056. doi: 10.1016/j.neuroimage.2011.10.015

Yang, C.-C., Barrós-Loscertales, A., Li, M., Pinazo, D., Borchardt, V., Ávila, C., et al. (2019). Alterations in brain structure and amplitude of low-frequency after 8 weeks of mindfulness meditation training in meditation-naive subjects. Sci. Rep. 9, 1-10. doi: 10.1038/s41598-019-47470-4

Zimmermann, J., Griffiths, J. D., and McIntosh, A. R. (2018). Unique mapping of structural and functional connectivity on cognition. J. Neurosci. 38, 9658-9667. doi: 10.1523/JNEUROSCI.0900-18.2018

Zuo, X.-N., and Xing, X.-X. (2014). Test-retest reliabilities of restingstate fMRI measurements in human brain functional connectomics: systems neuroscience perspective. Neurosci. Biobehav. Rev. 45, 100-118. doi: $10.1016 /$ j.neubiorev.2014.05.009

Conflict of Interest: The authors declare that the research was conducted in the absence of any commercial or financial relationships that could be construed as a potential conflict of interest.

Copyright (๑) 2021 RaviPrakash, Anwar, Biassou and Bagci. This is an open-access article distributed under the terms of the Creative Commons Attribution License (CC $B Y)$. The use, distribution or reproduction in other forums is permitted, provided the original author(s) and the copyright owner(s) are credited and that the original publication in this journal is cited, in accordance with accepted academic practice. No use, distribution or reproduction is permitted which does not comply with these terms. 